\title{
A pilot study of machine-learning based automated planning for primary brain tumours
}

Derek S. Tsang ${ }^{1 \dagger}$, Grace Tsui ${ }^{1 \dagger}$, Chris McIntosh¹, Thomas Purdie ${ }^{1}$, Glenn Bauman², Hitesh Dama', Normand Laperriere', Barbara-Ann Millar', David B. Shultz', Sameera Ahmed ${ }^{1}$, Mohammad Khandwala' and David C. Hodgson ${ }^{1 *}$

\begin{abstract}
Purpose: High-quality radiotherapy (RT) planning for children and young adults with primary brain tumours is essential to minimize the risk of late treatment effects. The feasibility of using automated machine-learning (ML) to aid RT planning in this population has not previously been studied.

Methods and materials: We developed a ML model that identifies learned relationships between image features and expected dose in a training set of 95 patients with a primary brain tumour treated with focal radiotherapy to a dose of 54 Gy in 30 fractions. This ML method was then used to create predicted dose distributions for 15 previouslytreated brain tumour patients across two institutions, as a testing set. Dosimetry to target volumes and organs-at-risk (OARs) were compared between the clinically-delivered (human-generated) plans versus the ML plans.

Results: The ML method was able to create deliverable plans in all 15 patients in the testing set. All ML plans were generated within 30 min of initiating planning. Planning target volume coverage with $95 \%$ of the prescription dose was attained in all plans. OAR doses were similar across most structures evaluated; mean doses to brain and left temporal lobe were lower in ML plans than manual plans (mean difference to left temporal, $-2.3 \mathrm{~Gy}, p=0.006$; mean differences to brain, $-1.3 \mathrm{~Gy}, p=0.017$ ), whereas mean doses to right cochlea and lenses were higher in ML plans (+ 1.6-2.2 Gy, $p<0.05$ for each).

Conclusions: Use of an automated ML method to aid RT planning for children and young adults with primary brain tumours is dosimetrically feasible and can be successfully used to create high-quality 54 Gy RT plans. Further evaluation after clinical implementation is planned.
\end{abstract}

Keywords: Machine-learning, Radiotherapy planning, Brain neoplasms

\section{Introduction}

Radiation therapy is an essential treatment for children and adults with brain tumours, but it can lead to important side effects including neurocognitive change, hearing loss and endocrinopathies. Designing RT treatments

*Correspondence: david.hodgson@rmp.uhn.ca

${ }^{\dagger}$ Derek S. Tsang and Grace Tsui have contributed equally to this work.

${ }^{1}$ Radiation Medicine Program, Princess Margaret Cancer Centre,

University Health Network, 610 University Avenue, Toronto, ON M5G 2M9, Canada

Full list of author information is available at the end of the article that maximize the likelihood of cure while minimizing side effects is crucial [1]. Although RT planning software has improved significantly in recent decades, the creation of RT plans for most tumour types is still dependent on a semi-manual iterative process of optimizing parameters to achieve an acceptable, inverse-planned RT dose distribution. This manual process of trial-and-error is operator-dependent and labor intensive, and while the resulting radiation dose distributions may meet specified clinical goals, they are not necessarily the optimal radiation plan for an individual patient. Automated planning original author(s) and the source, provide a link to the Creative Commons licence, and indicate if changes were made. The images or other third party material in this article are included in the article's Creative Commons licence, unless indicated otherwise in a credit line to the material. If material is not included in the article's Creative Commons licence and your intended use is not permitted by statutory regulation or exceeds the permitted use, you will need to obtain permission directly from the copyright holder. To view a copy of this licence, visit http://creativecommons.org/licenses/by/4.0/. The Creative Commons Public Domain Dedication waiver (http://creativeco mmons.org/publicdomain/zero/1.0/) applies to the data made available in this article, unless otherwise stated in a credit line to the data. 
is a method to overcome these limitations, and has been previously studied in patients with cervical cancer [2], prostate cancer [3], breast cancer [4], and lung cancer [5]. To our knowledge, no prior publication has described the successful use of automated planning to optimize radiation treatment of primary brain tumours.

In this study, we developed and evaluated an automated machine-learning RT planning method for children and adults with brain tumours. Deliverable ML-generated treatment plans were dosimetrically compared with human-generated plans that were delivered clinically.

\section{Materials and methods}

We performed an in silico dosimetry study to evaluate feasibility of ML planning for brain tumours, and the quality of the resulting RT plans. The study was approved by the relevant institutional Research Ethics Boards.

Details of ML model development have been described previously [6-8]. In brief, an atlas of clinically-treated photon plans was first created. Within the ML pipeline, contoured structures and computed tomography (CT) imaging features were extracted by the software. Imaging features describe the appearance and texture of the imaging dataset on a per-voxel basis, and account for differences in patient anatomical geometry (see Additional file 1: supplementary materials). The first ML component used atlas regression forests (ARFs) to associate image features with observed radiation dose. This process was repeated over each voxel for the entire CT dataset, on every case in the training dataset. A second component of the ML step was designed to ensure the accuracy of dose prediction by considering contextual information to the dose-per-voxel. Since each voxel's dose is not independent from the dose to adjacent voxels, the contextual dose links a voxel's dose to that of nearby voxels. A conditional random field (CRF) model was used to combine these individual voxel doses and generate predicted dose distributions that were spatially accurate and realistic over anatomic regions of interest. The trained ML model predicted the dose to targets and normal tissues for a novel patient case based on the learned relationships between imaging features and per-voxel dose by automatically identifying anatomically similar training cases. The predicted dose plans are then converted into clinically deliverable single-arc volumetric arc therapy (VMAT) plans using an inverse-planning optimization algorithm that minimizes the difference between the predicted and final dose, while ensuring technical beam delivery constraints are met, to create a deliverable plan.

We applied this approach to a training set of 95 consecutive brain tumour patients treated from July 2016 to August 2020 at a single institution (Fig. 1). Patients receiving focal treatment (no craniospinal radiotherapy component) to 54 Gy using VMAT for an intracranial brain tumour were eligible for inclusion. RT plans met evaluation criteria listed in Table 2.

Fifteen novel brain tumour patients clinically treated with 54 Gy in 30 fractions from July 2018 to November 2020 at two institutions were then re-planned with this ML model as a testing set (Fig. 1). These patients' novel planning CT images with target and organs-at-risk contours were input into the ML model for ML-plan

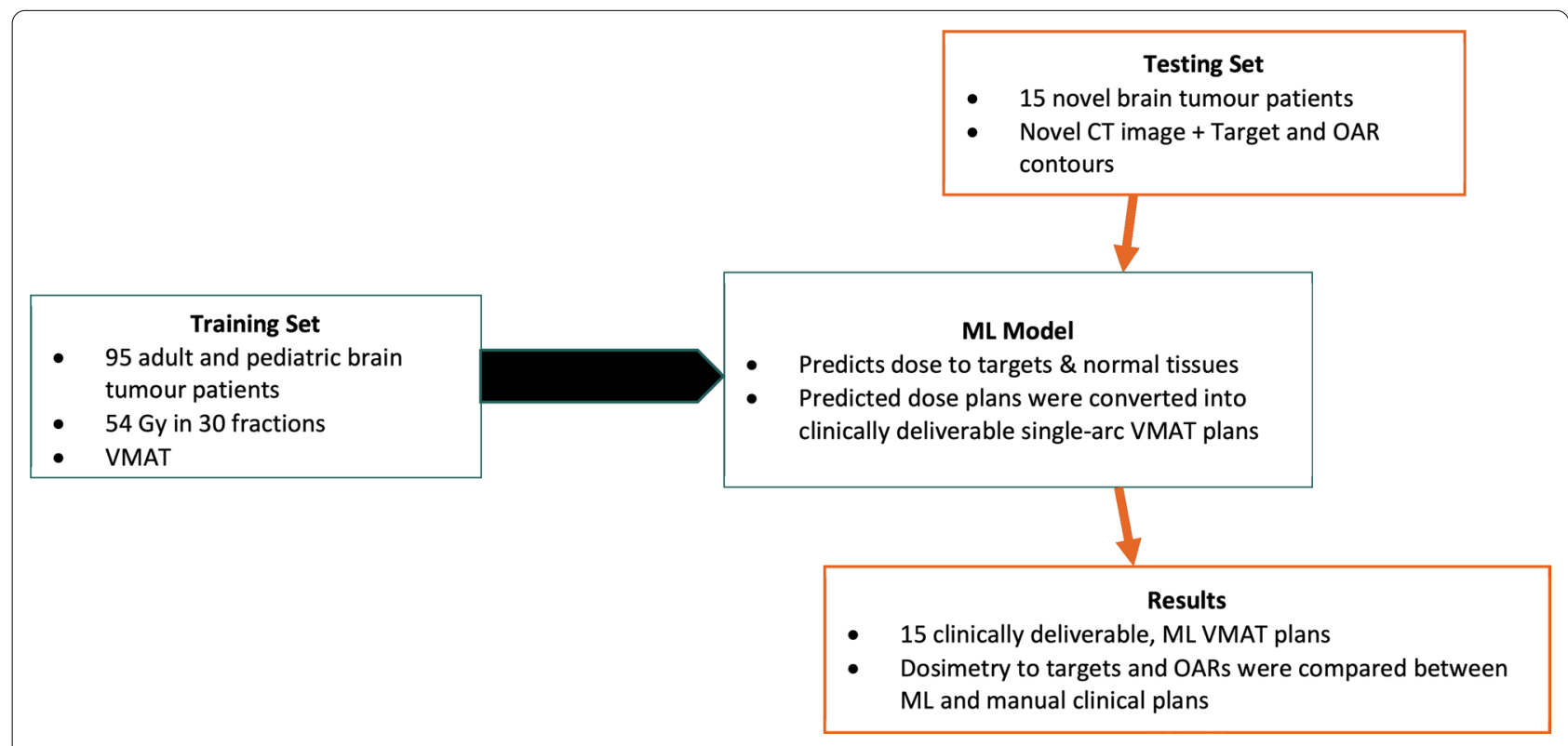

Fig. 1 Flow diagram of study data and planning workflow 
generation. Dosimetry to both target volumes and OARs was reviewed and compared with the manual, humangenerated plans that were delivered clinically. Target coverage, maximum doses to brainstem, optic chiasm, optic nerves, spinal cord, and mean doses to brain, hypothalamus, pituitary, cochlea, hippocampi, temporal lobes and parotids were evaluated and compared between ML and manual plans using paired $t$-tests.

\section{Results}

Details of our patient cohort are shown in Table 1. ML plans were successfully created for all 15 patients in the testing set. An example case is shown in Fig. 2, with representative manually-created clinical plan and the clinically-deliverable ML plan. All ML plans were generated within $30 \mathrm{~min}$ of initiating planning.

To evaluate ML plans in the testing set and compare with the manual plans, we first applied pre-specified plan evaluation criteria to both. The results of this comparison are shown in Table 2. Similar target coverage was observed in both ML and manual plans; at least $95 \%$ of PTV received $>51.3$ Gy (95\% of prescription) in all ML and manual plans. Maximum chiasm dose was $<54$ Gy in $14 \mathrm{ML}$ vs 15 manual plans; maximum brainstem dose was $<54$ Gy in all $15 \mathrm{ML}$ vs 13 manual plans.

We subsequently compared quantitative dose metrics to OARs, shown in Table 3. Maximum doses to brainstem, chiasm, each eye and optic nerve, spinal cord, and mean doses to right temporal lobe, left cochlea, each hippocampus, hypothalamus, parotid and pituitary were

Table 1 Patient characteristics in training and testing set

\begin{tabular}{lll}
\hline Characteristics & Training set $(\mathbf{n}=\mathbf{9 5})$ & Testing set $(\mathbf{n}=\mathbf{1 5})$ \\
\hline Age at RT, median (range) & $24(2,40)$ & $35(13,71)$ \\
Pediatric, age $<18(\%)$ & $38 \%$ & $13 \%$ \\
Female $(\%)$ & $49 \%$ & $33 \%$ \\
Diagnosis & & \\
Glioma & 62 & 9 \\
Meningioma & 6 & 3 \\
Ependymoma & 11 & 2 \\
Medulloblastoma* & 2 & - \\
Craniopharyngioma & 3 & - \\
Others & 11 & 1 \\
Tumor location & & \\
Supratentorial & 45 & 10 \\
Infratentorial & 50 & 5 \\
Tumor laterality & & 5 \\
Midline & 45 & 7 \\
Lateralized_left & 18 & 3 \\
Lateralized—right & 32 & \\
\hline
\end{tabular}

*54 Gy treatment for recurrence not statistically different between ML and manual plans ( $p>0.05$ for each). The maximum in-patient dose was not statistically different between ML and manual plans. Mean doses to brain and left temporal lobe were lower in ML plans than manual plans (mean difference to left temporal, $-2.3 \mathrm{~Gy}, p=0.006$; mean differences to brain, -1.3 Gy, $p=0.017)$, whereas mean doses to right cochlea and lenses were higher in ML plans (+1.6-2.2 Gy, $p<0.05$ for each).

\section{Discussion}

To our knowledge, this is the first study to demonstrate the feasibility of using ML planning to create high quality, clinically deliverable RT plans for patients with primary brain tumours. ML plans were comparable with manual plans with respect to their ability to meet a priori plan evaluation criteria, including target coverage. Quantitative dosimetry to OARs was similar in both approaches, indicating that ML plans would be suitable to use and implement for clinical treatments.

Previous studies have demonstrated promising results using fully automated RT planning for sites with limited inter-patient variation in anatomy such as prostate, breast and lung cancer. McIntosh et al., demonstrated the feasibility of the voxel-based approach used here to create deliverable prostate cancer RT plans [7, 9] and Duren-Koopman et al. developed personalized, scripted tangential and arc-based RT planning for patients requiring breast plus locoregional lymph nodes [4]. Similarly, Creemers et al. demonstrate excellent dosimetric characteristics of automated VMAT plans in non-small cell lung cancer, as compared with manual plans [10]. Among primary brain tumours, although the intracranial contents are similar between patients, the variation in brain tumour configuration, and the variable impact of tumor and surgery on normal CNS anatomy poses unique challenges that the ML method was able to overcome. This contrasts with prior studies of automated planning, which have primarily been applied to anatomically homogeneous targets.

When creating ML models, using high-quality RT plans in the training model is critical so that ML output is similarly high-quality [11]. In the present study, we applied strict dosimetric criteria for inclusion in the training set to ensure high-quality plans were included in the ML model. Our study is limited to use of homogeneous dose prescriptions (54 Gy); different training sets and models are likely needed for use with two-phase plans or other prescriptions because of differing dose-constraints on OARs. Clinical implementation to ensure continued feasibility is required; this process is ongoing at our institution. 


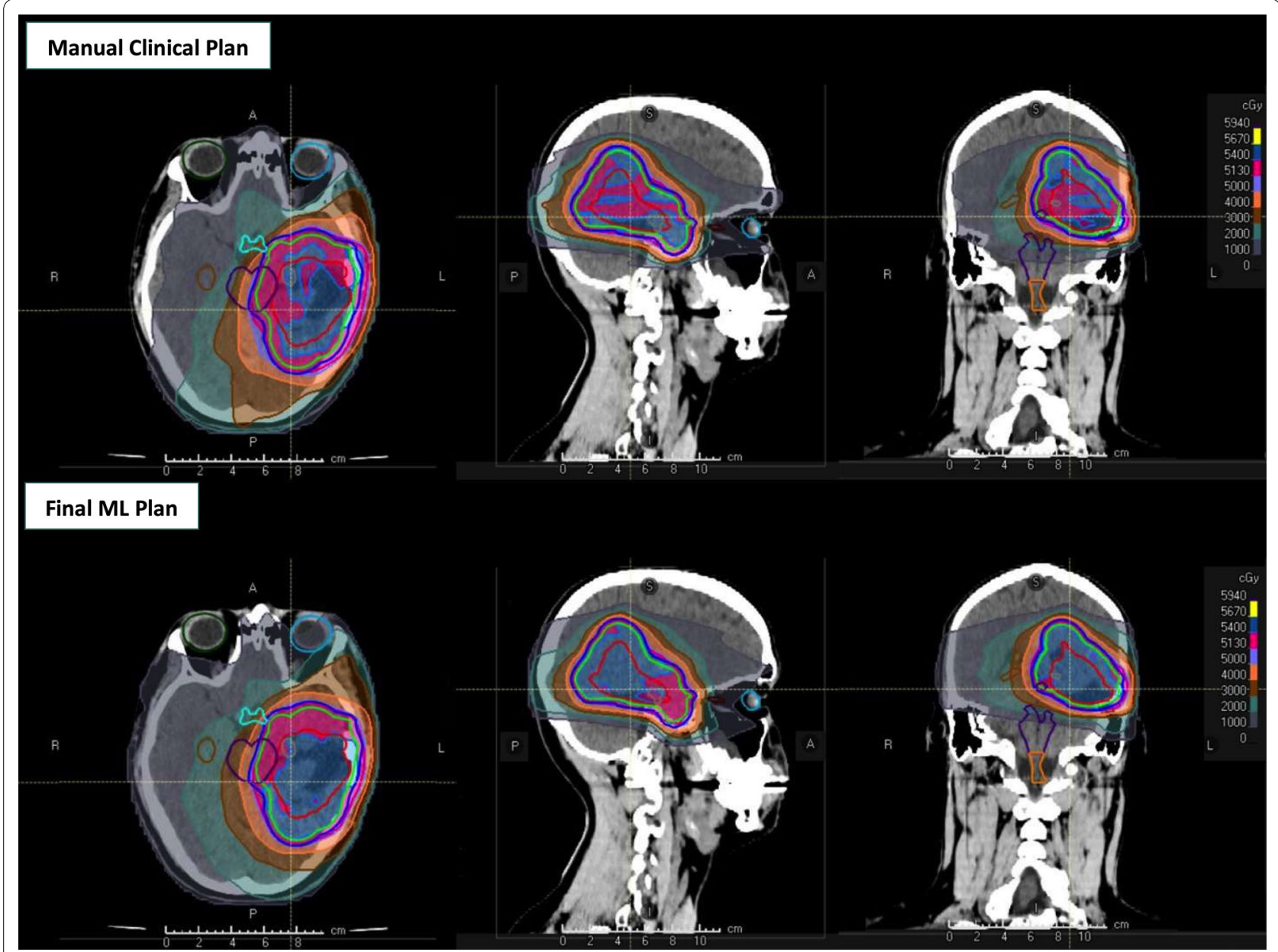

Fig. 2 Manually-created clinical plan on top row and final ML plan bottom row respectively. Axial, sagittal and coronal views are shown from left to right. Red, green and blue lines represent the gross tumor, clinical target and planning target volumes, respectively

Table 2 Evaluation criteria applied to manual and ML plans

\begin{tabular}{|c|c|c|c|c|c|}
\hline \multirow[t]{2}{*}{ Regions of interest } & \multirow[t]{2}{*}{$\mathrm{n}$} & \multirow[t]{2}{*}{ Criterion* } & \multicolumn{2}{|l|}{ Criterion met } & \multirow[t]{2}{*}{ Outcome } \\
\hline & & & Manual plans & ML plans & \\
\hline GTVp & 15 & D99 > 5130 cGy & 14 & 14 & Similar \\
\hline CTVp_5400 & 15 & D98 > 5130 cGy & 15 & 15 & Similar \\
\hline PTVp_5400 & 15 & D95 > 5130 cGy & 15 & 15 & Similar \\
\hline PTVp_5400 & 15 & Dmax <5670 cGy & 14 & 14 & Similar \\
\hline Brainstem & 15 & Dmax < 5400 cGy & 13 & 15 & ML better \\
\hline Chiasm & 15 & Dmax $<5400$ cGy & 15 & 14 & Manual better \\
\hline External & 15 & Dmax <5670 cGy & 14 & 14 & Similar \\
\hline Eye_L & 15 & Dmax $<4500$ cGy & 15 & 15 & Similar \\
\hline Eye_R & 15 & Dmax $<4500$ cGy & 15 & 15 & Similar \\
\hline Lens_L & 15 & Dmax < 750 cGy & 15 & 11 & Manual better \\
\hline Lens_R & 15 & Dmax < 750 cGy & 15 & 13 & Manual better \\
\hline OpticNrv_L & 15 & Dmax $<5400$ cGy & 15 & 15 & Similar \\
\hline OpticNrv_R & 15 & Dmax $<5400$ cGy & 15 & 15 & Similar \\
\hline SpinalCord & 15 & Dmax < 5400 cGy & 15 & 15 & Similar \\
\hline
\end{tabular}

${ }^{*}$ Dx represents the dose to $\mathrm{x} \%$ of the region of interest. Dmax represents the point max dose to the region of interest (i.e. a single voxel) 
Table 3 Summary of dose differences to OARs between ML and manual plans

\begin{tabular}{|c|c|c|c|c|c|c|c|c|c|}
\hline \multirow[t]{2}{*}{ Organs at risk } & \multirow[t]{2}{*}{$\mathbf{n}$} & \multirow[t]{2}{*}{ Criterion } & \multicolumn{2}{|c|}{ Mean values (cGy) } & \multicolumn{5}{|c|}{ Dose differences*, ML - manual (cGy) } \\
\hline & & & ML & Manual & Mean & $p$ value & Median & Maximum & Minimum \\
\hline Brainstem & 15 & Dmax $<5400$ cGy & 3820 & 3857 & -37 & 0.6984 & 8 & 601 & -1140 \\
\hline Chiasm & 15 & Dmax $<5400$ cGy & 3075 & 3267 & -192 & 0.3458 & 49 & 747 & -2051 \\
\hline External & 15 & Dmax $<5670$ cGy & 5599 & 5572 & 27 & 0.4019 & 45 & 220 & -238 \\
\hline Eye_L & 15 & Dmax $<4500$ cGy & 1417 & 1430 & -13 & 0.8923 & 26 & 549 & -614 \\
\hline Eye_R & 15 & Dmax $<4500$ cGy & 1211 & 1182 & 29 & 0.8388 & 56 & 988 & -927 \\
\hline Lens_L & 15 & Dmax $<750$ cGy & 586 & 369 & 217 & 0.0188 & 109 & 766 & -139 \\
\hline Lens_R & 15 & Dmax $<750$ cGy & 532 & 356 & 176 & 0.0204 & 177 & 814 & -218 \\
\hline OpticNrv_L & 15 & Dmax $<5400$ cGy & 2772 & 2701 & 71 & 0.5836 & 11 & 1130 & -890 \\
\hline OpticNrv_R & 15 & Dmax $<5400$ cGy & 2358 & 2342 & 16 & 0.8765 & 20 & 702 & -771 \\
\hline SpinalCord & 15 & Dmax $<5400$ cGy & 1271 & 1411 & -140 & 0.2440 & -9 & 104 & -1723 \\
\hline Brain & 15 & Dmean & 1476 & 1603 & -127 & 0.0172 & -62 & 96 & -615 \\
\hline Brain_Temporal_L & 15 & Dmean & 1758 & 1984 & -226 & 0.0056 & -155 & 69 & -854 \\
\hline Brain_Temporal_R & 15 & Dmean & 1324 & 1389 & -65 & 0.3871 & -3 & 426 & -662 \\
\hline Cochlea_L & 15 & Dmean & 1844 & 1555 & 289 & 0.2919 & 20 & 2850 & -2102 \\
\hline Cochlea_R & 15 & Dmean & 1579 & 1417 & 162 & 0.0276 & 31 & 703 & -77 \\
\hline Hippocampus_L & 14 & Dmean & 2005 & 2225 & -219 & 0.1441 & -90.5 & 642 & -1302 \\
\hline Hippocampus_R & 14 & Dmean & 1760 & 1729 & 31 & 0.8081 & 11.5 & 781 & -749 \\
\hline Hypothalamus & 15 & Dmean & 2214 & 2380 & -166 & 0.3638 & -121 & 804 & -1753 \\
\hline Parotid_L & 15 & Dmean & 145 & 273 & -128 & 0.1303 & -11 & 33 & -1177 \\
\hline Parotid_R & 15 & Dmean & 145 & 318 & -173 & 0.1051 & -14 & 18 & -1499 \\
\hline Pituitary & 15 & Dmean & 2392 & 2311 & 81 & 0.5159 & 40 & 846 & -1231 \\
\hline
\end{tabular}

Bolded text represents $p$ value $<0.05$

*Dose difference of an ROI is the dose in the ML plan minus dose in manual plan. Negative values indicate lower doses in the ML plan (better OAR sparing with ML plan)

The potential of ML model lies in its the ability to reliably create high-quality treatment plans that were not dependent on the training or skill of the medical dosimetrist, as well as rapid creation of reliable RT plans. This has important potential to improve access to high quality RT in small practices or middle-income countries where planning expertise may be limited [12]. Further, rapid RT planning is especially important for patients requiring urgent commencement of RT, such as in children with symptomatic brainstem glioma.

\section{Conclusions}

In conclusion, we developed and evaluated an automated machine-learning RT planning method for pediatric and adult brain tumour patients, and demonstrated the feasibility of rapidly generating clinically-deliverable ML plans that display consistent plan quality, as well as similar target coverage and OAR sparing as compared to human-generated plans used clinically. Clinical implementation of this ML treatment planning system is ongoing.

\section{Abbreviations}

RT: Radiotherapy; ML: Machine-learning; OARs: Organs-at-risk; PTV: Planning target volume; VMAT: Volumetric arc therapy; CT: Computed tomography;

ARFs: Atlas regression forests.

\section{Supplementary Information}

The online version contains supplementary material available at https://doi. org/10.1186/s13014-021-01967-3.

Additional file 1. Details of our automated planning platform and machine-learning pipeline.

Acknowledgements

Not applicable.

\section{Authors' contributions}

DCH and DST designed the study, and supervised all aspects of data collection, analysis, and interpretation. DST and GT wrote the manuscript. CM and TP made substantial contribution to the design of software used in this work. DST, GT, GB, HD, NL, BAM, DBS, AS, DCH, and MK collected study data. DST, $\mathrm{DCH}$, and GT analyzed study data. DCH, CM, TP, GB, NL, BAM, and DBS interpreted study data. All authors read, edited, and approved the final manuscript.

\section{Funding}

The presented work is funded by the Mount Sinai Hospital-University Health Network Academic Medical Organization Innovation Fund. 


\section{Availability of data and materials}

The datasets used and analyzed during the current study are available from the corresponding author on reasonable request.

\section{Declarations}

Ethics approval and consent to participate

This study was reviewed and approved by the Research Ethics Boards at University Health Network and Western University.

\section{Consent for publication}

\author{
Not applicable.
}

\section{Competing interests}

GT, GB, HD, NL, BAM, DBS, SA and MK declare that they have no competing interest. DST discloses that the institution has received Mount Sinai HospitalUniversity Health Network Academic Medical Organization Innovation Fund, in support of this work. CM has royalty bearing license of automated planning Al technology from RaySearch Laboratories, and patent "Method And System for Automated Quality Assurance And Automated Treatment Planning in Radiation Therapy". TP has received support from CIHR and NSERC, and has royalties from RaySearch Laboratories.

\section{Author details}

${ }^{1}$ Radiation Medicine Program, Princess Margaret Cancer Centre, University Health Network, 610 University Avenue, Toronto, ON M5G 2M9, Canada. ${ }^{2}$ London Regional Cancer Program, London, ON, Canada.

Received: 26 July 2021 Accepted: 15 December 2021

Published online: 06 January 2022

\section{References}

1. Merchant TE, Pollack IF, Loeffler JS. Brain tumors across the age spectrum: biology, therapy, and late effects. Semin Radiat Oncol. 2010;20:58-66.

2. Li N, et al. Highly efficient training, refinement, and validation of a knowledge-based planning quality-control system for radiation therapy clinical trials. Int J Radiat Oncol Biol Phys. 2017;97:164-72.

3. Heijmen $B$, et al. Fully automated, multi-criterial planning for volumetric modulated arc therapy - an international multi-center validation for prostate cancer. Radiother Oncol. 2018;128:343-8.

4. van Duren-Koopman MJ, et al. Personalized automated treatment planning for breast plus locoregional lymph nodes using hybrid rapidarc. Pract Radiat Oncol. 2018;8:332-41.

5. Vanderstraeten $B$, et al. Automated instead of manual treatment planning? A plan comparison based on dose-volume statistics and clinical preference. Int J Radiat Oncol Biol Phys. 2018;102:443-50.

6. McIntosh C, Purdie TG. Voxel-based dose prediction with multi-patient atlas selection for automated radiotherapy treatment planning. Phys Med Biol. 2017;62:415-31.

7. Mclntosh C, et al. Fully automated treatment planning for head and neck radiotherapy using a voxel-based dose prediction and dose mimicking method. Phys Med Biol. 2017;62:5926-44.

8. Mclntosh C, Purdie TG. Contextual atlas regression forests: multiple-atlasbased automated dose prediction in radiation therapy. IEEE Trans Med Imaging. 2016:35:1000-12.

9. Berlin A, et al. Clinical application of a novel voxel- and machine learningbased automated planning method for prostate volumetric arc radiation therapy. Int J Radiat Oncol Biol Phys. 2018;102:e533.

10. Creemers IHP, et al. Comparison of dose metrics between automated and manual radiotherapy planning for advanced stage non-small cell lung cancer with volumetric modulated arc therapy. Phys Imaging Radiat Oncol. 2019:9:92-6.

11. Boon IS, Au Yong TPT, Boon CS. Application of artificial intelligence (ai) in radiotherapy workflow: paradigm shift in precision radiotherapy using machine learning. Br J Radiol. 2019;92:20190716.

12. Tinoco $M$, et al. Rapidplan development of vmat plans for cervical cancer patients in low- and middle-income countries. Med Dosim. 2020;45:172-8.

\section{Publisher's Note}

Springer Nature remains neutral with regard to jurisdictional claims in published maps and institutional affiliations.
Ready to submit your research? Choose BMC and benefit from:

- fast, convenient online submission

- thorough peer review by experienced researchers in your field

- rapid publication on acceptance

- support for research data, including large and complex data types

- gold Open Access which fosters wider collaboration and increased citations

- maximum visibility for your research: over $100 \mathrm{M}$ website views per year

At BMC, research is always in progress.

Learn more biomedcentral.com/submissions 